\title{
A short note on Markov duality in multi-species higher spin stochastic vertex models
}

\author{
Jeffrey Kuan*
}

\begin{abstract}
We show that the multi-species higher spin stochastic vertex model, also called the $U_{q}\left(A_{n}^{(1)}\right)$ vertex model, satisfies a duality where the indicator function has the form $\left\{\eta_{[i, n]}^{x} \geq \xi_{[i, n]}^{x}\right\}$. In other words, for every particle in the $\xi$ configuration of species $i$ at vertex $x$, there must be a particle of species $j \geq i$ at vertex $x$ in the $\eta$ configuration. For these duality functions, the dual process has fewer particles than the original process, making it suitable for applications. The proof follows by applying charge reversal to previously discovered duality functions, which also results in open boundary conditions. As a special case, we recover the duality for the stochastic six vertex model recently found by Y. Lin.
\end{abstract}

Keywords: Markov duality; quantum groups; stochastic vertex models. MSC2020 subject classifications: 60 .

Submitted to ECP on November 18, 2019, final version accepted on June 25, 2021. Supersedes arXiv: 1911.01013v1.

\section{Introduction}

In [KMMO16], the authors construct stochastic $S$-matrices arising from the affine quantum group $U_{q}\left(A_{n}^{(1)}\right)$. In [Kua18], it is shown that the corresponding stochastic vertex model satisfies a Markov duality with its space reversal. The duality function had previously occurred as the duality function between multi-species $\operatorname{ASEP}(q, m / 2)$ and its space reversal [Kua17], where the function is only non-zero if

$$
\eta_{0}^{x}+\ldots+\eta_{n-i}^{x} \geq \xi_{i}^{x}+\ldots+\xi_{n}^{x}
$$

for all lattice site $x$ and species number $i \in\{1,2, \ldots, n\}$. Here, $\eta_{i}^{x} \in\{0,1, \ldots, m\}$ denotes the number of particles of species $i$ at the lattice site $x$ in a configuration denoted by $\eta$, and $\eta_{0}^{x}$ denotes the number of holes at lattice site $x$.

On the other hand, the duality function between multi-species $\operatorname{ASEP}(q, m / 2)$ and itself (non space-reversed) is non-zero if

$$
\eta_{i}^{x}+\ldots+\eta_{n}^{x} \geq \xi_{i}^{x}+\ldots+\xi_{n}^{x} .
$$

These types are duality functions are more suitable for applications of Markov duality, so it is natural to try to find these types of dualities for the stochastic $U_{q}\left(A_{n}^{(1)}\right)$ vertex models. However, for totally asymmetric models such as the stochastic vertex models, it

*Texas A\&M University, United States of America. E-mail: j kuan@math.tamu.edu 
is necessary to apply a space reversal (see the Remark after Theorem 2.5 of [Kua17]). In this paper, we show that the simultaneous inversions in the asymmetry parameter $q$ and spectral parameter $z$ will play the same role as a space reversal. This results in a new duality function for the stochastic $U_{q}\left(A_{n}^{(1)}\right)$ vertex model with open boundary conditions. For the case of the multi-species stochastic six vertex model, it generalizes the duality found in [Lin19] for the single-species stochastic six vertex model.

We note that there are duality functions for stochastic vertex models which do not have any indicator functions [CP16], [Lin20], but we will not discuss these here.

\section{Main results}

\subsection{Stochastic $S$-matrices}

First let us define some notation. Fix positive integers $l, m$ and $n$. Let $\mathcal{H}_{l}$ and $\mathcal{V}$ denote the sets

$$
\mathcal{H}_{l}=\left\{\left(\alpha_{0}, \ldots, \alpha_{n}\right): \alpha_{0}+\ldots+\alpha_{n}=l\right\}, \quad \mathcal{V}_{m}=\left\{\left(\beta_{0}, \ldots, \beta_{n}\right): \beta_{0}+\ldots+\beta_{n}=m\right\} .
$$

The $\mathcal{H}_{l}$ and $\mathcal{V}_{m}$ correspond to the horizontal and vertical lines of vertices, respectively. The $l$ and $m$ are the spins of the lines, that is, the level of the symmetric tensor representation.

For $i<j$, let $\alpha_{[i, j]}=\alpha_{i}+\ldots+\alpha_{j}$, and similarly for $\beta$. The paper [KMMO16] introduces a two-parameter family of stochastic ${ }^{1}$ matrices $S(q, z)$ whose rows and columns are indexed by $\mathcal{H}_{l} \times \mathcal{V}_{m}$. We let $S(q, z)_{\alpha \beta}^{\gamma \delta}$ denote the matrix entry with row $(\alpha, \beta)$ and column $(\gamma, \delta)$. The matrix $S(q, z)$ satisfies the conservation property that $S(q, z)$ is only nonzero if $\alpha+\beta=\gamma+\delta$. There are some explicit formulas for the matrix entries of $S(q, z)$ [BM16], but we will not use them here. See the end of this paper for a few examples.

We can represent $S(q, z)$ as vertex weights. Associate to each subscript $i \in\{0, \ldots, n\}$ a color. For $i<j$, we can consider particles of color $i$ to be lighter than particles of color $j$. In the example below, we have $l=1, m=2, \alpha=(0,1,0), \beta=(1,0,1), \gamma=(0,0,1), \delta=$ $(1,1,0)$; black is lighter than red which is lighter than green.

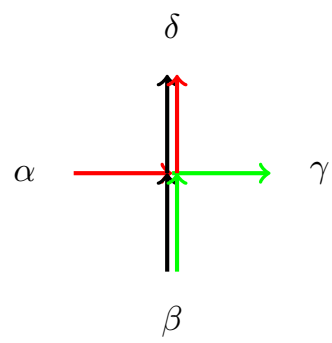

\subsection{Transfer matrices}

The stochastic $S$ matrices can be used to define an interacting particle system. Let $\bar{S}(q, z)$ denote the $\mathcal{V} \times \mathcal{H}_{l}$ by $\mathcal{H}_{l} \times \mathcal{V}$ matrix, where the entries are defined by

$$
\bar{S}(q, z)_{\beta \alpha}^{\gamma \delta}=S(q, z)_{\alpha \beta}^{\gamma \delta} \text {. }
$$

Given a positive integer $L$ and spectral parameters $\vec{z}=\left(z_{1}, \ldots, z_{L}\right)$, define the transfer matrix $\mathcal{T}_{L}$ to be the $\mathcal{V}_{m_{1}} \times \cdots \times \mathcal{V}_{m_{L}} \times \mathcal{H}_{l}$ by $\mathcal{H}_{l} \times \mathcal{V}_{m_{1}} \times \cdots \times \mathcal{V}_{m_{L}}$ matrix, given by the composition

$$
\mathcal{T}_{L}(q, \vec{z})=\bar{S}_{L-1, L}\left(q, z_{L}\right) \cdots \bar{S}_{12}\left(q, z_{2}\right) \bar{S}_{01}\left(q, z_{1}\right)
$$

\footnotetext{
${ }^{1}$ In this paper, we follow the common convention among mathematical physicists that a matrix is stochastic if its columns, rather than its rows, sum to 1 .
} 


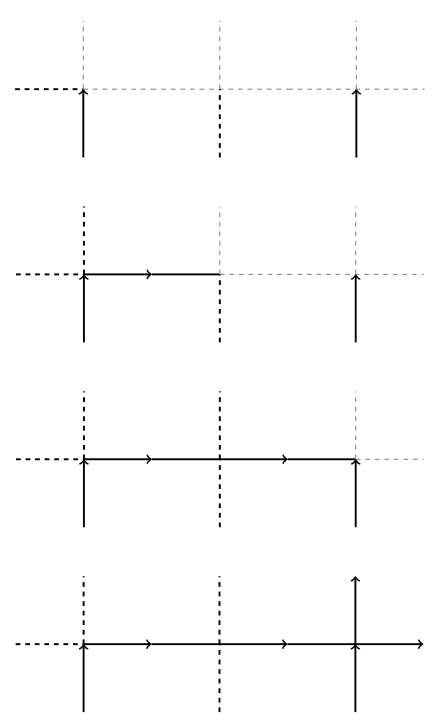

Figure 1: This example shows an update of the transfer matrix when $l=m=1$. This update has probability $z\left(q^{2}-1\right)(1-z)\left(q^{2}-z\right)^{-2}$; also see the examples in Section 3.

where

$$
\bar{S}_{j, j+1}(q, z):=\mathrm{Id}^{\otimes j} \otimes \bar{S}(q, z) \otimes \operatorname{Id}^{\otimes L-1-j}
$$

is a $\left(\mathcal{V}_{m_{1}} \times \cdots \mathcal{V}_{m_{j+1}} \times \mathcal{H}_{l} \times \mathcal{V}_{m_{j+2}} \times \cdots \times \mathcal{V}_{m_{L}}\right) \times\left(\mathcal{V}_{m_{1}} \times \cdots \mathcal{V}_{m_{j}} \times \mathcal{H}_{l} \times \mathcal{V}_{m_{j+1}} \times \cdots \times \mathcal{V}_{m_{L}}\right)$ matrix. Here, $m_{x}$ is the maximum number of particles that may occupy lattice site $x$.

These transfer matrices can be viewed as the transition probabilities for a discretetime totally asymmetric particle system, either on the infinite line or on a finite lattice. See Figure 1 for an example. We use the bold greek symbols $\boldsymbol{\eta}, \boldsymbol{\xi}$ to denote elements of $\mathcal{V}_{m_{1}} \times \cdots \times \mathcal{V}_{m_{L}}$, where $\eta^{x} \in \mathcal{V}_{m_{x}}$ for $1 \leq x \leq L$. By a slight abuse of notation, we allow $L$ to equal infinity, so that $x$ can take values in $\mathbb{Z}$ or $\mathbb{Z}_{>0}$. Let

$$
|\boldsymbol{\xi}|=\sum_{x} \sum_{i=1}^{n} \xi_{i}^{x}
$$

and if $|\boldsymbol{\xi}|<\infty$ then we say that $\boldsymbol{\xi}$ has finitely many particles.

The stochastic matrix of transition probabilities for the discrete-time totally asymmetric particle system is then denoted $\mathcal{P}\left(\boldsymbol{\eta}, \boldsymbol{\eta}^{\prime}\right)$, and is defined by

$$
\mathcal{P}\left(\boldsymbol{\eta}, \boldsymbol{\eta}^{\prime}\right)=\sum_{\gamma \in \mathcal{H}_{l}} \mathcal{T}_{L}\left((\boldsymbol{\eta}, \gamma),\left(\mathbf{0}, \boldsymbol{\eta}^{\prime}\right)\right),
$$

where the notation in $q, \vec{z}$ has been suppressed. The sum over $\xi$ is a sum over all possible sets of particles that exit the lattice $\{1, \ldots, L\}$.

We also let $\mathcal{P}_{\text {rev }}$ denote the space-reversed version of $\mathcal{P}$. In $\mathcal{P}$, the particles jump to the right, whereas in $\mathcal{P}_{\text {rev }}$, the particles jump to the left. More formally, let $\widetilde{S}(q, z)$ denote the $\mathcal{H}_{l} \times \mathcal{V}$ by $\mathcal{V} \times \mathcal{H}_{l}$ matrix, where the entries are defined by

$$
\widetilde{S}(q, z)_{\alpha \beta}^{\delta \gamma}=S(q, z)_{\alpha \beta}^{\gamma \delta}
$$

Define the transfer matrix $\widetilde{\mathcal{T}}_{L}$ to be the $\mathcal{H}_{l} \times \mathcal{V}_{m_{1}} \times \cdots \times \mathcal{V}_{m_{L}}$ by $\mathcal{V}_{m_{1}} \times \cdots \times \mathcal{V}_{m_{L}} \times \mathcal{H}_{l}$ matrix, given by the composition

$$
\widetilde{\mathcal{T}}_{L}(q, \vec{z})=\widetilde{S}_{01}\left(q, z_{L}\right) \cdots \widetilde{S}_{L-1, L-2}\left(q, z_{2}\right) \widetilde{S}_{L-1, L}\left(q, z_{1}\right)
$$


where as before

$$
\widetilde{S}_{j, j+1}(q, z):=\mathrm{Id}^{\otimes j} \otimes \widetilde{S}(q, z) \otimes \mathrm{Id}^{\otimes L-1-j}
$$

is a $\left(\mathcal{V}_{m_{1}} \times \cdots \mathcal{V}_{m_{j+1}} \times \mathcal{H}_{l} \times \mathcal{V}_{m_{j+2}} \times \cdots \times \mathcal{V}_{m_{L}}\right) \times\left(\mathcal{V}_{m_{1}} \times \cdots \mathcal{V}_{m_{j}} \times \mathcal{H}_{l} \times \mathcal{V}_{m_{j+1}} \times \cdots \times \mathcal{V}_{m_{L}}\right)$ matrix. Then $\mathcal{P}_{\text {rev }}$ is defined by

$$
\mathcal{P}_{\text {rev }}\left(\boldsymbol{\eta}, \boldsymbol{\eta}^{\prime}\right)=\sum_{\gamma \in \mathcal{H}_{l}} \tilde{\mathcal{T}}_{L}\left((\gamma, \boldsymbol{\eta}),\left(\boldsymbol{\eta}^{\prime}, \mathbf{0}\right)\right)
$$

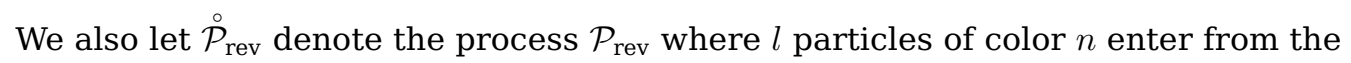
right boundary at every time step. In other words,

$$
\left.\stackrel{\circ}{\mathcal{P}}_{\text {rev }}\left(\boldsymbol{\eta}, \boldsymbol{\eta}^{\prime}\right)=\sum_{\gamma \in \mathcal{H}_{l}} \tilde{\mathcal{T}}_{L}(\gamma, \boldsymbol{\eta}),\left(\boldsymbol{\eta}^{\prime}, \mathbf{l}\right)\right),
$$

where 1 consists of $l$ particles of color $n$. Such boundary conditions had appeared previously in [Bor17], in the single-species case. See also [BW] for the multi-species case.

If the dependence on the asymmetry parameter $q$ and the spectral parameter $z$ needs to be specified, write $\mathcal{P}(q, \vec{z})$ or $\mathcal{P}_{\text {rev }}(q, \vec{z})$ or $\stackrel{\circ}{\mathcal{P}}_{\text {rev }}(q, \vec{z})$.

Let $D$ be the duality function

$$
D(\xi, \eta)=\prod_{x}\left[\eta_{0}^{x}\right]_{q}^{!} \cdots\left[\eta_{n}^{x}\right]_{q}^{!} \prod_{i=1}^{n}\left(\begin{array}{c}
\eta_{[i-1, n]}^{x}-\xi_{[i, n]}^{x} \\
\eta_{i-1}^{x}
\end{array}\right)_{q} \cdot q^{-\xi_{i}^{x}\left(\eta_{[0, i-1]}^{x}+\sum_{z>x} 2 \eta_{[0, i-1]}^{z}\right)},
$$

where $[k]_{q}$ is the $q$-deformed integer

$$
[k]_{q}=\frac{q^{k}-q^{-k}}{q-q^{-1}}
$$

$[k]_{q}^{!}$is the $q$-deformed factorial $[1]_{q}[2]_{q} \cdots[k]_{q}$, and

$$
\left(\begin{array}{l}
n \\
k
\end{array}\right)_{q}=\frac{[n]_{q}^{!}}{[k]_{q}^{!}[n-k]_{q}^{!}}
$$

is the $q$-deformed binomial. If $n<k$ we set $\left(\begin{array}{l}n \\ k\end{array}\right)_{q}=0$. In particular, $D(\xi, \eta)$ is only nonzero if $\eta_{[j, n]}^{x} \geq \xi_{[j, n]}^{x}$ for all $x$ and $j$. Note that when all $m_{x}=m$, then $D$ can also be written as

$$
D(\xi, \eta)=\mathrm{const} \cdot \prod_{x}\left[\eta_{0}^{x}\right]_{q}^{!} \cdots\left[\eta_{n}^{x}\right]_{q}^{!} \prod_{i=1}^{n}\left(\begin{array}{c}
\eta_{[i-1, n]}^{x}-\xi_{[i, n]}^{x} \\
\eta_{i-1}^{x}
\end{array}\right)_{q} \cdot q^{2 m x \xi_{i}^{x}+\xi_{i}^{x}\left(\eta_{[i, n]}^{x}+\sum_{z>x} 2 \eta_{[i, n]}^{z}\right)},
$$

where const is a constant that does not change under the dynamics. To see this, write

$$
\eta_{[0, i-1]}^{y}=m-\eta_{[i, n]}^{y}
$$

for all $y$ and $i$. Plugging this into the original definition of $D$ and setting const to be

$$
\text { const }=q^{2(L+1) m|\boldsymbol{\xi}|}
$$

shows the alternative form of $D$.

Similarly, define

$$
\tilde{D}(\xi, \eta)=\prod_{x}\left[\eta_{0}^{x}\right]_{q}^{!} \cdots\left[\eta_{n}^{x}\right]_{q}^{!} \prod_{i=1}^{n}\left(\begin{array}{c}
\eta_{[i-1, n]}^{x}-\xi_{[i, n]}^{x} \\
\eta_{i-1}^{x}
\end{array}\right)_{q} \cdot q^{\xi_{i}^{x}\left(\eta_{[i, n]}^{x}+\sum_{z>x} 2 \eta_{[i, n]}^{z}\right)} .
$$

The duality result is stated below as an intertwining; this intertwining is the definition of Markov duality. The ${ }^{*}$ denotes the transposition of the matrix, and $\vec{z}^{-1}$ denotes $\left(z_{1}^{-1}, \ldots, z_{L}^{-1}\right)$. 
Theorem 2.1. For any $q$ and $\vec{z}$,

$$
\mathcal{P}^{*}(q, \vec{z}) D=D \stackrel{\circ}{\mathcal{P}}_{\text {rev }}\left(q^{-1}, \vec{z}^{-1}\right) .
$$

Proof. We start with an intertwining of the $S$-matrices, which previously appeared but had not been applied to duality. Let $\Pi_{*}$ denote the matrix that reverses the order of the colors. In other words, $\Pi_{\mathcal{H}_{l}}$ and $\Pi_{\mathcal{V}}$ denote the permutation matrices with entries

$$
\Pi_{\mathcal{H}_{l}}(\alpha, \gamma)=\left\{\begin{array}{l}
1, \text { if } \alpha_{i}=\gamma_{n-i} \text { for all } i \in\{0,1, \ldots, n\} \\
0, \text { else }
\end{array}\right.
$$

and

$$
\Pi_{\mathcal{V}}(\beta, \delta)=\left\{\begin{array}{l}
1, \text { if } \beta_{i}=\delta_{n-i} \text { for all } i \in\{0,1, \ldots, n\} \\
0, \text { else }
\end{array}\right.
$$

Proposition 3.7 of [Kua18] implies that

$$
\left(\Pi_{\mathcal{H}_{l}} \otimes \Pi_{\mathcal{V}}\right) \circ S(q, z) \circ\left(\Pi_{\mathcal{H}_{l}} \otimes \Pi_{\mathcal{V}}\right)=S\left(q^{-1}, z^{-1}\right) .
$$

Alternatively, one can check directly when $l=m=1$, and then use the fusion relation in Theorem 3.4 of [Kua18].

One consequence of (2.1) is that

$$
\left(\Pi_{\mathcal{V}} \otimes \Pi_{\mathcal{H}_{l}}\right) \circ \bar{S}(q, z) \circ\left(\Pi_{\mathcal{H}_{l}} \otimes \Pi_{\mathcal{V}}\right)=\bar{S}\left(q^{-1}, z^{-1}\right) .
$$

Letting $\bar{\Pi}^{(L)}$ denote $\Pi_{\mathcal{H}_{l}} \otimes \Pi_{\mathcal{V}_{m_{1}}} \otimes \cdots \otimes \Pi_{\mathcal{V}_{m_{L}}}$ and $\Pi^{(L)}$ denote $\Pi_{\mathcal{V}_{m_{1}}} \otimes \cdots \otimes \Pi_{\mathcal{V}_{m_{L}}} \otimes \Pi_{\mathcal{H}_{l}}$, the previous equation implies

$$
\tilde{\mathcal{T}}_{L}\left(q^{-1}, \vec{z}^{-1}\right) \circ \Pi^{(L)}=\bar{\Pi}^{(L)} \circ \tilde{\mathcal{T}}_{L}(q, \vec{z}) .
$$

Letting $\Pi$ is defined on $\mathcal{V}_{m_{1}} \times \cdots \times \mathcal{V}_{m_{L}}$, we have that

$$
\begin{aligned}
\left.\left(\stackrel{\circ}{\mathcal{P}} \text { rev }^{-1}, q^{-1}\right) \circ \Pi\right)\left(\boldsymbol{\eta}, \boldsymbol{\eta}^{\prime}\right) & =\sum_{\gamma \in \mathcal{H}_{l}}\left(\tilde{\mathcal{T}}_{L}\left(q^{-1}, \vec{z}^{-1}\right)\right)\left((\gamma, \boldsymbol{\eta}),\left(\Pi_{\mathcal{H}_{l}} \boldsymbol{\eta}^{\prime}, \mathbf{l}\right)\right) \\
& =\sum_{\gamma \in \mathcal{H}_{l}}\left(\tilde{\mathcal{T}}_{L}\left(q^{-1}, \vec{z}^{-1}\right) \circ \Pi^{(L)}\right)\left((\gamma, \boldsymbol{\eta}),\left(\boldsymbol{\eta}^{\prime}, \mathbf{0}\right)\right) \\
& \stackrel{(2.2)}{=} \sum_{\gamma \in \mathcal{H}_{l}}\left(\bar{\Pi}^{(L)} \circ \tilde{\mathcal{T}}_{L}(q, \vec{z})\right)\left((\gamma, \boldsymbol{\eta}),\left(\boldsymbol{\eta}^{\prime}, \mathbf{0}\right)\right) \\
& =\sum_{\gamma \in \mathcal{H}_{l}}\left(\tilde{\mathcal{T}}_{L}(q, \vec{z})\right)\left(\left(\Pi_{\mathcal{H}_{l}} \gamma, \Pi \boldsymbol{\eta}\right),\left(\boldsymbol{\eta}^{\prime}, \mathbf{0}\right)\right) \\
& =\left(\Pi \circ \mathcal{P}_{\text {rev }}(q, \vec{z})\right)\left(\boldsymbol{\eta}, \boldsymbol{\eta}^{\prime}\right) .
\end{aligned}
$$

Thus, the conclusion is that

$$
\left.\stackrel{\circ}{\mathcal{P}} \text { rev }^{-1}, q^{-1}\right) \circ \Pi=\Pi \circ \mathcal{P}_{\text {rev }}(q, \vec{z}) .
$$

For the single-species $(n=1)$ case, (2.3) reduces to Proposition 5.1 of [CP16].

The previous duality result (Theorem $4.10^{2}$ of [Kua18]) says that

$$
\mathcal{P}^{*}(q, \vec{z}) \circ D \Pi=D \Pi \circ \mathcal{P}_{\text {rev }}(q, \vec{z}) .
$$

\footnotetext{
${ }^{2}$ There is a typo in the paper [Kua18]. Equations (16) and (17) of [Kua18], which define DП, cite [Kua17] but switch the $\eta$ and the $\xi$. This can also be corrected by reversing the direction of the jumps in $\eta$ and $\xi$ (which corresponds to switching $\eta$ and $\xi$ ), as noted by [Lin19]. In any case, we will not use the explicit expression of $D \Pi$ here.
} 
To see why (2.4) follows from Theorem 4.10, start with the duality function in Theorem 4.10. The paper [Kua18] uses different notation than here, which amounts to substituting $i \mapsto n+1-i$. Next, substitute $m_{x}-\eta_{[i+1, n]}^{x}=\eta_{[0, i]}$. Next, the application of $\Pi$ replaces every $\eta_{i}$ with $\eta_{n-i}$. Finally, make the substitution $i \mapsto n+1-i$ in the index of the product.

As noted in Remark 5 of [Kua], intertwinings of the form (2.3) can be used to produce new dualities from old ones. Because $\Pi=\Pi^{*}$ and $\Pi^{2}=$ id, plugging (2.3) into (2.4) implies that

$$
\mathcal{P}^{*}(q, \vec{z}) D \Pi=D \stackrel{\circ}{\text { rev }}\left(q^{-1}, \vec{z}^{-1}\right) \Pi .
$$

Multiplying by $\Pi$ on both sides shows the theorem.

Remark 2.2. If the lattice is the infinite line $\mathbb{Z}$ and all $m_{x}$ are equal to 1 , then the theorem can be written as

$$
\mathcal{P}^{*}(q, \vec{z}) D=q^{2 l|\boldsymbol{\xi}|} D \mathcal{P}_{\text {rev }}\left(q^{-1}, \vec{z}^{-1}\right) .
$$

This is because the $l$ particles entering from the right still contribute to the duality, even as the lattice size grows to infinity. In other words, the full-line duality is obtained by considering a finite lattice $\{-L, \ldots, L\}$ and then taking $L \rightarrow \infty$. For large values of $L$, the $l$ particles that enter the lattice will not interact with the particles in $\xi$. However, they contribute a factor of $q^{2 l}$ to the duality, which remains even in the limit. See the first example in section 3 for an illustrative example.

If, on the other hand, the lattice is $\{\ldots,-2,-1\}$, then we have a duality between a stochastic vertex model with particles jumping to the left and allowed to enter at -1 , and a stochastic vertex model with particles jumping to the right and allowed to exit from -1 . Similar types of boundary conditions have occurred in duality results; see [GKRV09], [CGR19], [Kua]. The second example in section 3 also illustrates a special case of this type of boundary condition.

Remark 2.3. In the notation of [CP16], the duality function is

$$
D(\xi, \eta)=\mathrm{const} \cdot \prod_{x}\left[\eta_{0}^{x}\right]_{q^{1 / 2}}^{!} \cdots\left[\eta_{n}^{x}\right]_{q^{1 / 2}}^{!} \prod_{i=1}^{n}\left(\begin{array}{c}
\eta_{[i-1, n]}^{x}-\xi_{[i, n]}^{x} \\
\eta_{i-1}^{x}
\end{array}\right)_{q^{1 / 2}} \cdot q^{m x \xi_{i}^{x}+\xi_{i}^{x}\left(\frac{1}{2} \eta_{[i, n]}^{x}+\sum_{z>x} \eta_{[i, n]}^{z}\right)} .
$$

Note that the duality function does not depend on $z$ or $l$. If the size of the lattice is taken to infinity, then the term const needs to be scaled with the size of the lattice to ensure that the duality function is nonzero and finite. If all $m_{x}$ equal 1 , as is the case in the following theorem, no scaling is needed as the size of the lattice is taken to infinity.

Theorem 2.4. Suppose that the lattice is the infinite line $\mathbb{Z}$, and all $m_{x}$ and $l$ equal 1. Then

$$
\mathcal{P}^{*}\left(q^{-1}, \vec{z}^{-1}\right) \tilde{D}=\tilde{D} \mathcal{P}_{\text {rev }}\left(q^{-1}, \vec{z}^{-1}\right) .
$$

Proof. Now assume the conditions in the second duality result. Let $S$ denote the shift operator defined by

$$
S(\boldsymbol{\eta}, \boldsymbol{\xi})= \begin{cases}1, & \text { if } \eta^{x}=\xi^{x+1} \text { for all } x \\ 0, & \text { else. }\end{cases}
$$

We have that $S^{-1}=S^{*}$, and the translation invariance says that

$$
S^{-1} \mathcal{P} S=\mathcal{P}, \quad S^{-1} \mathcal{P}_{\text {rev }} S=\mathcal{P}_{\text {rev }} .
$$

Additionally, from the expressions for $\tilde{D}$ it is immediate that

$$
S^{-1} \tilde{D} S=\tilde{D} .
$$


Short note on duality in multi-species vertex model

Furthermore,

$$
S^{-1} D S=q^{-2|\boldsymbol{\xi}|} D
$$

due to the $\prod_{x} \prod_{i} q^{2 m x \xi_{x}^{i}}$ term. Let $G$ be the diagonal matrix with entries

$$
G(\boldsymbol{\xi}, \boldsymbol{\xi})=\prod_{x \in \mathbb{Z}} \prod_{i=1}^{n} q^{-2 m x \xi_{i}^{x}}
$$

From the definitions of $D$ and $\tilde{D}$, the matrix $G$ relates $D$ and $\tilde{D}$ via

$$
\tilde{D}=\text { const } \cdot G D \text {. }
$$

Once we show

$$
S G S^{*} \mathcal{P}^{*}(q, \vec{z}) G^{-1}=\mathcal{P}^{*}\left(q^{-1}, \vec{z}^{-1}\right),
$$

this proves the result. Indeed, assuming (2.5),

$$
\begin{aligned}
\mathcal{P}^{*}(q, \vec{z}) D & =D \stackrel{\circ}{\operatorname{rev}}\left(q^{-1}, \vec{z}^{-1}\right) \\
\Longrightarrow S G S^{*} \mathcal{P}^{*}(q, \vec{z}) G^{-1} G D & =S G S^{*} D \stackrel{\circ}{\mathcal{P}}_{\text {rev }}\left(q^{-1}, \vec{z}^{-1}\right) \\
\Longrightarrow \mathcal{P}^{*}\left(q^{-1}, \vec{z}^{-1}\right) \tilde{D} & =q^{-2|\boldsymbol{\xi}|} S \tilde{D} S^{*} \stackrel{\circ}{\mathcal{P}}_{\text {rev }}\left(q^{-1}, \vec{z}^{-1}\right) .
\end{aligned}
$$

The transition matrix $\stackrel{\circ}{\mathcal{P}}$ has a particle entering the lattice at each update, which produces a term $q^{2|\boldsymbol{\xi}|}$ (cf. Remark 1), thus finally yielding $\mathcal{P}^{*}\left(q^{-1}, \vec{z}^{-1}\right) \tilde{D}=\tilde{D} \mathcal{P}_{\text {rev }}\left(q^{-1}, \vec{z}^{-1}\right)$.

To show (2.5), first write it in the form

$$
\mathcal{P}\left(q^{-1}, \vec{z}^{-1}\right)=G^{-1} \mathcal{P}(q, \vec{z}) S G S^{*}
$$

Set

$$
b_{1}=\frac{q^{2}(1-z)}{q^{2}-z}, \quad b_{2}=\frac{1-z}{q^{2}-z}
$$

and note that $b_{1} / b_{2}=q^{2}$, and the simultaneous inversions $q \mapsto q^{-1}, z \mapsto z^{-1}$ switches $b_{1}$ and $b_{2}$. After this inversion, the weight $b_{1}$ is the "west-east" weight and $b_{2}$ is the "north-south" weight; initially $b_{1}$ is the "north-south" weight and $b_{2}$ is the "west-east" weight. Every matrix entry of $\mathcal{P}\left(q^{-1}, \vec{z}^{-1}\right)$ is of the form

$$
b_{2}^{A_{1}} b_{1}^{A_{2}}\left(\left(1-b_{1}\right)\left(1-b_{2}\right)\right)^{A_{3}}
$$

for some non-negative integers $A_{1}, A_{2}, A_{3}$. Proceed by induction on the value of $A_{2}$.

Suppose $A_{2}=0$. Define a block in the configuration $\xi$ to be an interval $\{x, x+1, \ldots, y\}$ where $\xi^{x-1} \neq \xi^{x}=\xi^{x+1}=\cdots=\xi^{y} \neq \xi^{y+1}$. The condition that $A_{2}=0$ means that every particle either stayed put, or was part of a block that jumped one step to the right (i.e., if a particle jumps two steps to the right, then there is at least one $b_{1}$ vertex, so $A_{2}>0$ ). The number of particles that stayed put is $A_{1}$, and the number of jumping blocks is $A_{3}$. Thus, the corresponding matrix entry of $G^{-1} \mathcal{P}(q, \vec{z}) S G S^{*}$ is

$$
b_{1}^{A_{1}}\left(\left(1-b_{2}\right)\left(1-b_{1}\right)\right)^{A_{3}} \cdot\left(\frac{b_{2}}{b_{1}}\right)^{A_{1}} 1^{A_{3}},
$$

which equals $b_{2}^{A_{1}}\left(\left(1-b_{1}\right)\left(1-b_{2}\right)\right)^{A_{3}}$.

Now suppose we know (2.5) for every matrix entry satisfying $A_{2}=r$. Let $\boldsymbol{\eta}, \boldsymbol{\xi}$ be particle configurations such that the $(\boldsymbol{\eta}, \boldsymbol{\xi})$ entry of $\mathcal{P}\left(q^{-1}, \vec{z}^{-1}\right)$ equals

$$
b_{2}^{A_{1}} b_{1}^{r+1}\left(\left(1-b_{1}\right)\left(1-b_{2}\right)\right)^{A_{3}} \text {. }
$$


Then there exists a $\boldsymbol{\zeta}$ such that the $(\boldsymbol{\zeta}, \boldsymbol{\xi})$ entry of $\mathcal{P}\left(q^{-1}, \vec{z}^{-1}\right)$ equals

$$
b_{2}^{C_{1}} b_{1}^{r}\left(\left(1-b_{1}\right)\left(1-b_{2}\right)\right)^{C_{3}}
$$

for some $C_{1}, C_{3}$. The configuration $\zeta$ can be constructed from $\boldsymbol{\eta}$ by taking a particle at lattice site $y$ and moving it to lattice site $x-1$ (where $x \leq y$ ), and $\{x, x+1, \ldots, y\}$ is a block in $\boldsymbol{\eta}$. Then $C_{1}-A_{1}=y-x$. The $(\boldsymbol{\zeta}, \boldsymbol{\xi})$ entry of $G^{-1} \mathcal{P}(q, \vec{z}) S G S^{*}$ is, by definition,

$$
[\mathcal{P}(q, \vec{z})](\boldsymbol{\zeta}, \boldsymbol{\xi}) \frac{G\left(\boldsymbol{\xi}^{+}, \boldsymbol{\xi}^{+}\right)}{G(\boldsymbol{\zeta}, \boldsymbol{\zeta})}
$$

where the superscript ${ }^{+}$denotes the particle configuration obtained by shifting every particle one lattice site to the right. By the induction hypothesis,

$$
[\mathcal{P}(q, \vec{z})](\boldsymbol{\zeta}, \boldsymbol{\xi}) \frac{G\left(\boldsymbol{\xi}^{+}, \boldsymbol{\xi}^{+}\right)}{G(\boldsymbol{\zeta}, \boldsymbol{\zeta})}=\left[\mathcal{P}\left(q^{-1}, \vec{z}^{-1}\right)\right](\boldsymbol{\zeta}, \boldsymbol{\xi}) .
$$

By construction,

$$
G(\boldsymbol{\zeta}, \boldsymbol{\zeta})=G(\boldsymbol{\eta}, \boldsymbol{\eta}) q^{2(y-x+1)}=G(\boldsymbol{\eta}, \boldsymbol{\eta})\left(\frac{b_{1}}{b_{2}}\right)^{C_{1}-A_{1}+1} .
$$

So by translation invariance,

$$
b_{1}^{C_{1}} b_{2}^{r}\left(\left(1-b_{2}\right)\left(1-b_{1}\right)\right)^{C_{3}} \frac{G\left(\boldsymbol{\xi}^{+}, \boldsymbol{\xi}^{+}\right)}{G(\boldsymbol{\eta}, \boldsymbol{\eta})}\left(\frac{b_{2}}{b_{1}}\right)^{C_{1}-A_{1}+1}=b_{2}^{C_{1}} b_{1}^{r}\left(\left(1-b_{1}\right)\left(1-b_{2}\right)\right)^{C_{3}},
$$

which simplifies to

$$
b_{1}^{A_{1}} b_{2}^{r+1}\left(\left(1-b_{2}\right)\left(1-b_{1}\right)\right)^{C_{3}} \frac{G\left(\boldsymbol{\xi}^{+}, \boldsymbol{\xi}^{+}\right)}{G(\boldsymbol{\eta}, \boldsymbol{\eta})}=b_{2}^{A_{1}} b_{1}^{r+1}\left(\left(1-b_{1}\right)\left(1-b_{2}\right)\right)^{C_{3}},
$$

Multiplying both sides by $\left(\left(1-b_{1}\right)\left(1-b_{2}\right)\right)^{A_{3}-C_{3}}$, we arrive at

$$
[\mathcal{P}(q, \vec{z})](\boldsymbol{\eta}, \boldsymbol{\xi}) \frac{G\left(\boldsymbol{\xi}^{+}, \boldsymbol{\xi}^{+}\right)}{G(\boldsymbol{\eta}, \boldsymbol{\eta})}=\left[\mathcal{P}\left(q^{-1}, \vec{z}^{-1}\right)\right](\boldsymbol{\eta}, \boldsymbol{\xi}),
$$

as needed.

Remark 2.5. In the single-species ( $n=1)$ case of Theorem 2.4, the stochastic vertex model reduces to the stochastic six vertex model. After replacing $q$ by $q^{1 / 2}$ and swapping the roles of left and right jumps, the duality reduces to the duality in [Lin19].

Remark 2.6. In the degeneration of the stochastic six vertex model to ASEP, we recover a duality for ASEP. Heuristically, as $z \rightarrow 1$,

$$
\begin{gathered}
\mathcal{P}(q, z) \approx S-L_{\mathrm{ASEP}\left(q^{2}, 1\right)} S(z-1)+\mathcal{O}\left((z-1)^{2}\right), \\
\mathcal{P}\left(q^{-1}, z^{-1}\right) \approx S^{*}-L_{\mathrm{ASEP}\left(1, q^{2}\right)} S^{*}(z-1)+\mathcal{O}\left((z-1)^{2}\right) .
\end{gathered}
$$

Here, $L_{\mathrm{ASEP}(l, r)}$ is the generator of ASEP with left jump rates $l$ and right jump rates $r$. The two dualities in this paper then become the Schütz ASEP duality [Sch97] and the ASEP duality in Corollary 4.7(c) from [Kua]. Note that the latter duality function had already occurred in [Sch97], using time reversal rather than space reversal. The relation (2.5) then reduces to the intertwining in the proof of Theorem 4.6(b) of [Kua].

Remark 2.7. As mentioned in the appendix of [CP16], the degeneration of the stochastic six vertex model to ASEP does not work for higher spin $(m>1)$ models, because nonnegativity fails to hold. Thus, the $m=1$ case can be regarded as a unique case; (2.5) does not hold in general, for example. 


\section{Examples}

When $l=1$, there is a simple expression for $S(z)$ from (25) of [Kua18]. Note that in the notation of [Kua18], particle colors are written as $\left(\eta_{1}, \ldots, \eta_{n+1}\right)$, where $\eta_{n+1}$ are the holes. The notation can be matched with the replacement $\eta_{i} \mapsto n+1-\eta_{i}$.

For $0 \leq j \leq n$, let $\epsilon_{j} \in \mathcal{H}_{l}$ denote the vector $(0, \ldots, 0,1,0, \ldots, 0)$, where the 1 is located the $j$ th index. For any $0 \leq i \leq j \leq n$, let $\alpha_{[i, j]}=\alpha_{i}+\alpha_{i+1}+\cdots+\alpha_{j}$.

$$
\left(q^{m+1}-z\right) S(z)_{\epsilon_{j}, \beta}^{\epsilon_{k}, \delta}=1_{\left\{\epsilon_{j}+\beta=\epsilon_{k}+\delta\right\}} \times \begin{cases}q^{2 \beta_{[k, n]}-m+1}\left(1-q^{-2 \beta_{k}+m-1} z\right), & \text { if } k=j \\ -q^{2 \beta_{[k+1, n]}-m+1}\left(1-q^{2 \beta_{k}}\right), & \text { if } k<j \\ -q^{2 \beta_{[k+1, n]}} z\left(1-q^{2 \beta_{k}}\right), & \text { if } k>j\end{cases}
$$

The stochastic matrices from Definition 2.1 of [CP16] uses the notations $\alpha, q, I$, which are related to the notations here by the substitutions $m \rightarrow I, q \rightarrow q^{1 / 2}, z \rightarrow-\alpha q \mu^{-1}$, where $\mu=q^{-m}$; see also Proposition 3.10 and Appendix A.2 of [Kua18] for a matching of the notations when $l>1$.

Below are the four nontrivial vertex weights for $l=m=1$ and $n=1$; the number of arrows coming in from the southern direction is equal to $\beta_{1}$ :

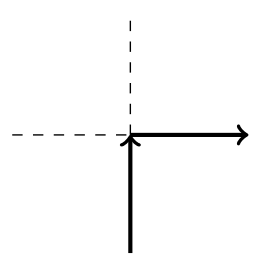

$\frac{z\left(q^{2}-1\right)}{q^{2}-z}$

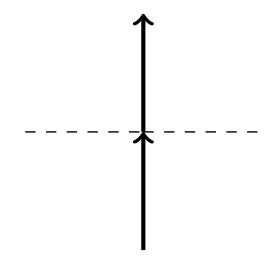

$\frac{q^{2}(1-z)}{q^{2}-z}$

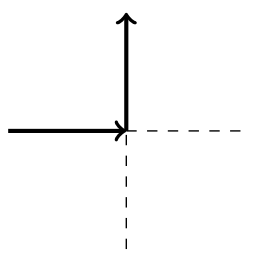

$\frac{q^{2}-1}{q^{2}-z}$

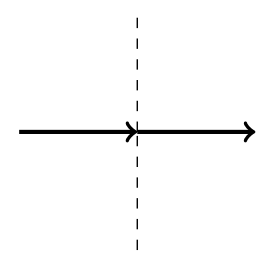

$\frac{1-z}{q^{2}-z}$

In the image below, the image shows $\mathcal{T}_{L}^{\text {rev }}$, which has weight $\left(q^{2}-z\right)^{-3}\left(-z\left(1-q^{2}\right)\right) \cdot(1-$ $z) \cdot\left(-\left(1-q^{2}\right)\right)$.

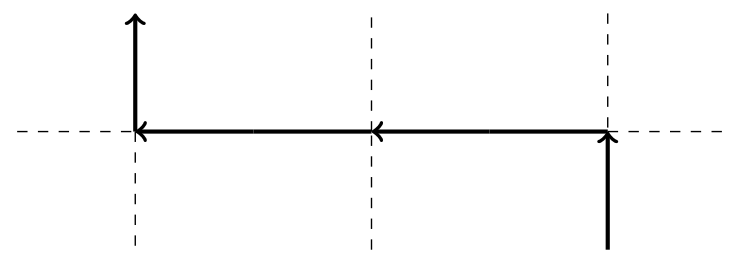

Below are the eight nontrivial vertex weights when $l=1, m=2$ and $n=1$; again the number of arrows coming in from the southern direction is equal to $\beta_{1}$ : 
Short note on duality in multi-species vertex model

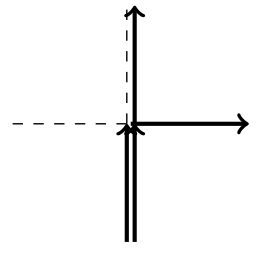

$\frac{z\left(q^{4}-1\right)}{q^{3}-z}$

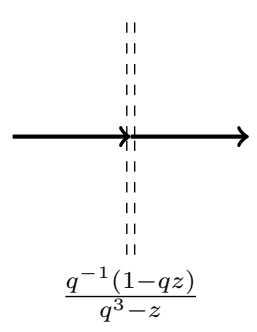

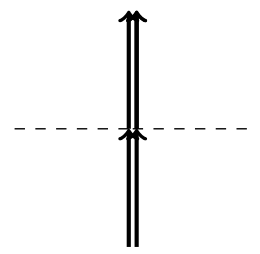

$\frac{q^{3}(1-q z)}{q^{3}-z}$

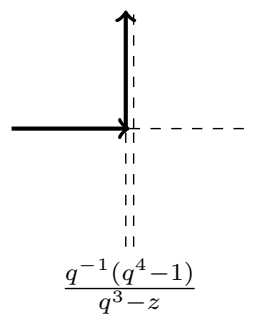

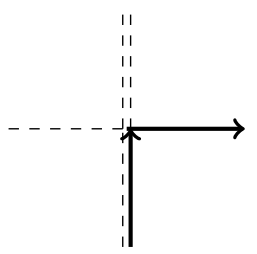

$\frac{z\left(q^{2}-1\right)}{q^{3}-z}$

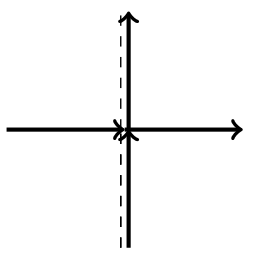

$\frac{q\left(1-q^{-1} z\right)}{q^{3}-z}$

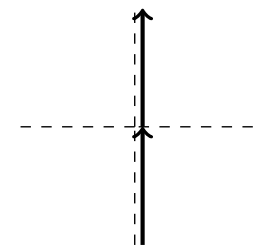

$\frac{q^{3}\left(1-q^{-1} z\right)}{q^{3}-z}$

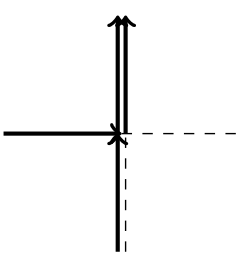

$\frac{q\left(q^{2}-1\right)}{q^{3}-z}$

We work out some examples; assume below that $n=1$ :

- Let us verify that on the infinite line,

$$
\left[\mathcal{P}^{*}(q, \vec{z}) D\right](\boldsymbol{\xi}, \boldsymbol{\eta})=\left[D \stackrel{\circ}{\mathcal{P}}_{\text {rev }}\left(q^{-1}, \vec{z}^{-1}\right)\right](\boldsymbol{\xi}, \boldsymbol{\eta})
$$

when $\boldsymbol{\xi}$ consists solely of one particle at lattice site 0 and $\boldsymbol{\eta}$ consists of $k$ particles at lattice site 0 , and $m_{0}=m$. The left-hand-side simplifies as

$$
\left[\mathcal{P}^{*}(q, \vec{z})\right](\boldsymbol{\xi}, \boldsymbol{\xi}) D(\boldsymbol{\xi}, \boldsymbol{\eta})=\frac{q^{m+1}\left(1-q^{-(m-1)} z\right)}{q^{m+1}-z} \cdot \frac{q^{k}-q^{-k}}{q-q^{-1}} q^{k} .
$$

The right-hand-side has two terms, one corresponding to when all particles in $\eta$ stay still, and the other corresponding to when one particle jumps:

$$
\begin{aligned}
& {\left[D \stackrel{\circ}{\text { rev }}\left(q^{-1}, \vec{z}^{-1}\right)\right](\boldsymbol{\xi}, \boldsymbol{\eta}) } \\
= & \left(\frac{q^{-(m+1)}\left(1-q^{m-2 k+1} z^{-1}\right)}{q^{-(m+1)}-z^{-1}} \frac{q^{k}-q^{-k}}{q-q^{-1}} q^{k}+\frac{z^{-1}\left(q^{-2 k}-1\right)}{q^{-(m+1)}-z^{-1}} \frac{q^{k-1}-q^{-k+1}}{q-q^{-1}} q^{k-1}\right) q^{2} .
\end{aligned}
$$

The factor of $q^{2}$ comes from the particle entering from the right. From a direct calculation, both sides equal

$$
-\frac{q^{k+1}\left(q^{k}-q^{-k}\right)\left(-q^{m}+q z\right)}{\left(q-q^{-1}\right)\left(q^{1+m}-z\right)} .
$$

- Let us verify that on $\{\ldots,-3,-2,-1\}$,

$$
\left[\mathcal{P}^{*}(q, \vec{z}) D\right](\boldsymbol{\xi}, \boldsymbol{\eta})=\left[D \stackrel{\circ}{\mathcal{P}}_{\text {rev }}\left(q^{-1}, \vec{z}^{-1}\right)\right](\boldsymbol{\xi}, \boldsymbol{\eta})
$$

where $\boldsymbol{\xi}$ consists solely of one particle at lattice site $-r$ and $\boldsymbol{\eta}$ is empty. The left-hand-side is

$$
\frac{z\left(q^{2}-1\right)}{\left(q^{m_{-r}+1}-z\right)} \cdot \frac{q^{-m_{-r+1}+1}-z}{q^{m_{-r+1}+1}-z} \cdots \frac{q^{-m_{-1}+1}-z}{q^{m_{-1}+1}-z},
$$


where the right-hand-side is

$$
\begin{aligned}
& \left.\frac{q^{-m_{-1}+1}-z}{q^{m_{-1}+1}-z} \cdots \frac{q^{-m_{-r+1}+1}-z}{q^{m_{-r+1}+1}-z} \cdot \frac{q^{-m_{-r}+1}\left(q^{2 m_{-r}}-1\right)}{q^{m_{-r}+1}-z}\right|_{z \mapsto z^{-1}, q \mapsto q^{-1}} \cdot D(\boldsymbol{\xi}, \boldsymbol{\xi}) \\
& =\frac{q^{2 m_{-1}}\left(q^{-m_{-1}+1}-z\right)}{q^{m_{-1}+1}-z} \cdots \frac{q^{2 m_{-r+1}}\left(q^{-m_{-r+1}+1}-z\right)}{q^{m_{-r+1}+1}-z} \cdot \frac{z q^{m_{-r}-1} \cdot q^{-m_{-r}+1}\left(q^{2 m_{-r}}-1\right)}{q^{m_{-r}+1}-z} \\
& \quad \times \frac{1}{\left[m_{-r}\right]_{q}} q^{-\left(m_{-r}-1\right)-\sum_{s=-r+1}^{-1} 2 m_{s}}
\end{aligned}
$$

which equals the left-hand-side.

\section{References}

[BM16] Gary Bosnjak and Vladimir V Mangazeev. Construction of $R$-matrices for symmetric tensor representations related to $U_{q}\left(\widehat{s l_{n}}\right)$. Journal of Physics A: Mathematical and Theoretical, 49(49):495204, nov 2016. MR3584389

[Bor17] Alexei Borodin. On a family of symmetric rational functions. Advances in Mathematics, 306:973-1018, 2017. MR3581324

[BW] Alexei Borodin and Michael Wheeler. Coloured stochastic vertex models and their spectral theory. arXiv:1808.01866v1.

[CGR19] Gioia Carinci, Cristian Giardinà, and Frank Redig. Consistent particle systems and duality. arXiv e-prints, page arXiv:1907.10583, Jul 2019. MR4320949

[CP16] Ivan Corwin and Leonid Petrov. Stochastic higher spin vertex models on the line. Communications in Mathematical Physics, 343(2):651-700, 2016. MR3477349

[GKRV09] Cristian Giardiná, Jorge Kurchan, Frank Redig, and Kiamars Vafayi. Duality and hidden symmetries in interacting particle systems. J. Stat. Phys, 135:25-55, 2009. MR2505724

[KMMO16] Atsuo Kuniba, Vladimir V. Mangazeev, Shouya Maruyama, and Masato Okado. Stochastic $R$ matrix for $U_{q}\left(A_{n}^{(1)}\right)$. Nuclear Physics B, 913:248-277, 2016. MR3575300

[Kua] Jeffrey Kuan. Stochastic fusion of interacting particle systems and duality functions. arXiv:1908.02359v1.

[Kua17] Jeffrey Kuan. A multi-species $\operatorname{ASEP}(q, j)$ and $q$-TAZRP with stochastic duality. International Mathematics Research Notices, 2018(17):5378-5416, 2017. MR3862123

[Kua18] Jeffrey Kuan. An algebraic construction of duality functions for the stochastic $U_{q}\left(A_{n}^{(1)}\right)$ vertex model and its degenerations. Communications in Mathematical Physics, 359(1):121-187, Apr 2018. MR3781448

[Lin19] Yier Lin. Markov duality for stochastic six vertex model. Electron. Commun. Probab., 24:17 pp., 2019. MR4029436

[Lin20] Yier Lin. KPZ equation limit of stochastic higher spin vertex model. Math Phys Anal Geom, 23(1), 2020. MR4046044

[Sch97] Gunter M Schütz. Duality relations for asymmetric exclusion processes. Journal of Statistical Physics, 86(5/6):1265-1287, 1997. MR1450767

Acknowledgments. The author thanks Yier Lin for helpful discussions. 


\section{Electronic Journal of Probability Electronic Communications in Probability}

\section{Advantages of publishing in EJP-ECP}

- Very high standards

- Free for authors, free for readers

- Quick publication (no backlog)

- Secure publication $\left(\mathrm{LOCKSS}^{1}\right)$

- Easy interface (EJMS²)

\section{Economical model of EJP-ECP}

- Non profit, sponsored by $\mathrm{IMS}^{3}, \mathrm{BS}^{4}$, ProjectEuclid ${ }^{5}$

- Purely electronic

\section{Help keep the journal free and vigorous}

- Donate to the IMS open access fund ${ }^{6}$ (click here to donate!)

- Submit your best articles to EJP-ECP

- Choose EJP-ECP over for-profit journals

\footnotetext{
${ }^{1}$ LOCKSS: Lots of Copies Keep Stuff Safe http://www. lockss.org/

${ }^{2}$ EJMS: Electronic Journal Management System http://www.vtex.lt/en/ejms.html

${ }^{3}$ IMS: Institute of Mathematical Statistics http://www.imstat.org/

${ }^{4}$ BS: Bernoulli Society http://www. bernoulli-society.org/

${ }^{5}$ Project Euclid: https://projecteuclid.org/

${ }^{6}$ IMS Open Access Fund: http://www.imstat.org/publications/open.htm
} 